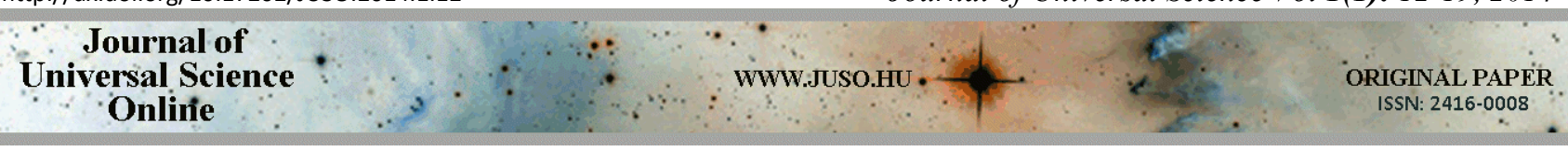

\title{
Molecular modeling of DDT's and it's major metabolites adsorption in the interlaminar space of montmorillonite
}

\author{
János ECKER ${ }^{1, *}$ and László FÜLÖP ${ }^{1}$
}

\author{
${ }^{1}$ Department of Chemistry and Biochemistry, Szent István University, Páter K.u.1., Gödöllő, H-2103 Hungary \\ *Corresponding Author; e-mail: Ecker.Janos.Mail@gmail.com
}

Received: 22 September 2014 / Revised: 3 November 2014 / Accepted: 11 December 2014

Keywords: adsorption, DDT, force fields, molecular dynamics, montmorillonite

Abbreviations:

DDT - 1,1,1-trichloro-2,2-bis( $p$-chlorophenyl)ethane

DDD - 1-chloro-4-[2,2-dichloro-1-(4-chlorophenyl)ethyl]benzene

DDE - 1,1-bis-(4-chloropenyl)-2,2-dichloroethane

\begin{abstract}
A simple adsorption mechanism of the organochloride insecticide DDT and it's most common metabolites DDD and DDE in the interlaminar layer of montmorillonite mineral is discussed in this paper, based on the results of molecular modeling. A model of a simple fragment of the montmorillonite crystal with the interlaminar layer was created and used. The interlaminar layer contained water as a solvent and the ionic forms of sodium or calcium as exchangeable cations along with the molecules of DDT/DDD/DDE. The molecular interactions were simulated by force fields and the results of the simulation could be explained by the physical characteristics of the models which were studied with bioinformatics tools as well. Based on the observed mechanisms, the adsorption of DDT and it's metabolites in the interlaminar layer of montmorillonite is possible and it is partly responsible for the persistent nature of these insecticides.
\end{abstract}

\section{Introduction}

DDT and the two metabolites (DDD, DDE) are still widely exist in soils and in other environmental elements with high concentrations $[16,21,23]$ due to their persistence, long-distance transportation (mostly by atmospheric transport) and bioaccumulation [22]. The DDD and DDE metabolites come from the dehydrochlorination of DDT and their physical and chemical properties are similar [18]. The dehydrochlorination and other types of catalyzed reactions that result in a change of the chemical structure are possible by enzymes, expressed by resistant insects and some species of bacteria and fungi [13, 19, 20], and a photooxidation mechanism of DDT and DDE has also been discovered $[1,11,15]$.

Beside these chemical transformations, the decreasing tendency of DDT concentrations in soil is a result of volatilization, water runoff and the harvesting of plants that have absorbed the insecticide [7].

The persistence of DDT and it's metabolites is not only determined by the hydrophobic character, structure and the limited biodegradability of these molecules. In soils, adsorption characteristics of organic pollutants are especially affected by the 


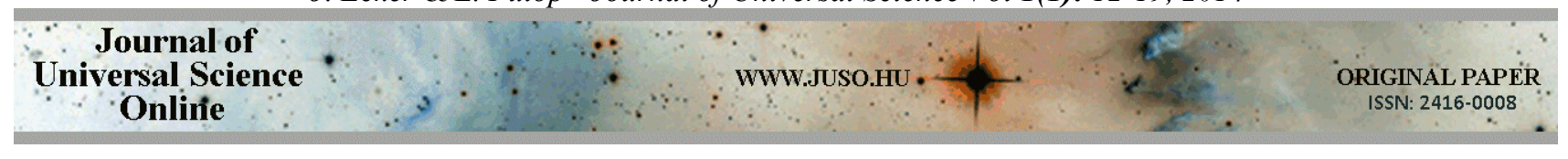

physicochemical properties and material compositions of the soil, for instance the decrease/increase in $\mathrm{pH}$ values [14] and contents of organic matters (mainly humic substances) [5]. Cheng [4] concluded that smaller soil particles has greater adsorption capacity to DDT and the adsorption is related to the composition and origin of organic matter and minerals.

The structure of montmorillonite (along with other minerals) was first proposed by Pauling (1930) by the X-ray diffraction method. The composition of montmorillonite is $\left[(\mathrm{Na}, \mathrm{Ca})(\mathrm{Al}, \mathrm{Mg})_{2} \mathrm{Si}_{4} \mathrm{O}_{10}(\mathrm{OH})_{2} \mathrm{XnH}_{2} \mathrm{O}\right]$, so chemically it is a hydrated sodium calcium aluminium magnesium silicate hydroxide. The unit layer is composed of three tetrahedral-octahedral-tetrahedral sheets. The center of the tetrahedron is $\mathrm{Si}$, it can be

\section{Material and methods}

\section{Applications}

Two applications were used during the study. The Avogadro software (v1.1.1) was a suitable tool to build the molecular structures atom by atom or from fragments and visualize their interactions. This application can render interactive, three-dimensional images and it is possible to optimize the structures geometrically and analyse the chemical and molecular data, as described by Hanwell [8]. Optimizing the structures of the "hand-drawn" molecules is an essential procedure to get the adequate bond lengths and angles. Two of Avogadro's force fields were used for

\section{The Montmorillonite Model}

The montmorillonite layer used in the simulations was finalized by modifying the structure of the pyrophyllite. This mineral has only $\mathrm{Si}$ in the tetrahedral and $\mathrm{Al}$ in the octahedral positions without substitutions, so it was an ideal intermediate model for the further studies. In the montmorillonite, the tetrahedral and octahedral sheets are substituted by Al. In the center of the octahedron there is $\mathrm{Al}$ and that can be substituted by $\mathrm{Mg}$ or $\mathrm{Fe}$ [3]. Due to it's adsorptive character, montmorillonite is widely used as an adsorbent in a variety of fields for example the elimination of heavy metals from contaminated water [12].

The adsorption of various chemicals is possible on the surface of the layer and in the interlaminar layer as well. Simple cations can be adsorbed on the surface or in the interlaminar layer by the montmorillonite's cation exchange capacity [2] but beside these phenomena which are controlled by electrostatic interactions, the van der Waals forces and the hydrogen bonds are also responsible for the adsorption, as described by Hendricks [9] and in the molecular simulation studies of Karaborni [10].

structure optimization but every force field has it's limitations. The Merck Molecular Force Field (MMFF94) is ideal for visualize the molecular interactions but it cannot be used with every element of the periodic table. The Universal Force Field (UFF) can be used across the entire periodic table.

The Marvin Sketch application (v14.8.18, developed by ChemAxon Ltd., Hungary) has many features which can be used to analyze the various characteristics of the models (e.g., charges, protonation, solubility) previously built and optimized by Avogadro.

repeating, so the size of the crystal is theoretically not limited. In the end it was duplicated and optimized with the UFF.

In the following step the covalent bonds between the aluminium and oxygen atoms were removed and the total amount of Al-s (48) in the two crystals were substituted by $\mathrm{Mg}$ and $\mathrm{Fe}$ atoms in random arrangement and 


$\begin{aligned} & \text { Journal of } \\ & \text { Universal Science } \\ & \text { Online }\end{aligned} \quad \cdots, \cdots \cdots$

MMFF94 was used. Despite these metals are atoms in appearance, without the covalent bonds the program handles them as di- and trivalent cations $\left(\mathrm{Mg}^{2+}, \mathrm{Fe}^{3+}\right)$. Substitutions in

\section{The Complete Model}

The final model contained all of the components for which molecular interaction studies were planned. The interlaminar space of the montmorillonite was filled with water molecules (solvent), DDT/DDD/DDE molecules and $\mathrm{Na}^{+} / \mathrm{Ca}^{2+}$ (exchangeable

\section{Results and discussions}

The optimized (UFF) fragment of the montmorillonite's starting model is shown in Fig. 1. Some substitutions in the tetrahedral (Al) and octahedral (Fe) positions have been the tetrahedral centers ( $\mathrm{Si}$ to $\mathrm{Al}$ ) were not performed, because the MMFF94 cannot be run with aluminium.

cations). The models of the pesticides were based on the chemical structures published in the work of Faroon \& Harris [6] and they were inserted into the interlaminar space randomly. In the final step the optimization by the MMFF94 was performed.



Fig. 1. Fragment of the montmorillonite starting model, based on the pyrophyllite with an empty interlaminar space, optimized by UFF (Avogadro 1.1.1). The pictures are rotated 90 degrees relative to each other. The colors indicate the following atoms: $\mathrm{O}$ (red), $\mathrm{Si}$ (blue), $\mathrm{Al}$ (grey), $\mathrm{Fe}$ (orange). 


Journal of
Universal Science
Online

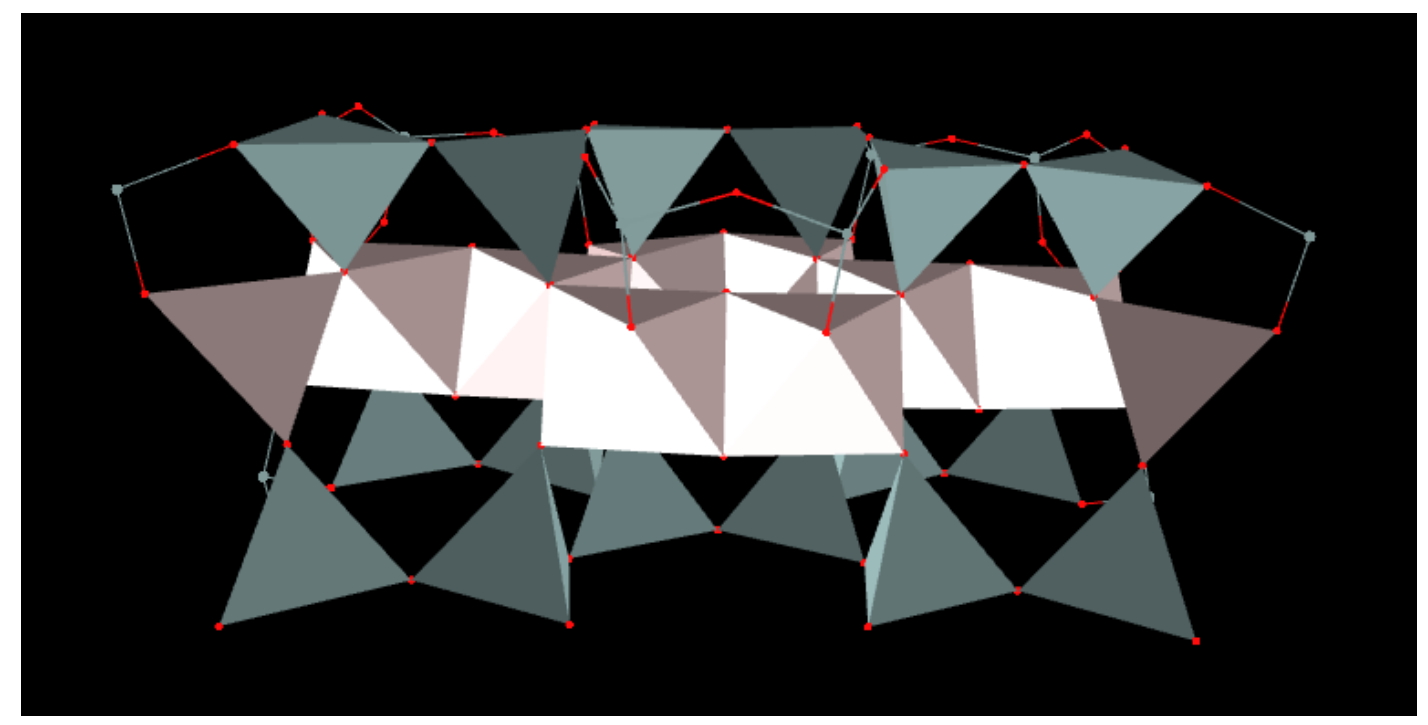

Fig. 2. Tetrahedral and octahedral sheets in the montmorillonite fragment (Avogadro 1.1.1).

In Fig. 2. the tetrahedral and octahedral sheets are visualized, the geometry of the layer is clearly visible. The final layer which is compatible with MMFF94 is an extended and modified version of the model shown in Fig. 1. The final structure used in the simulations is shown in Fig. 3.

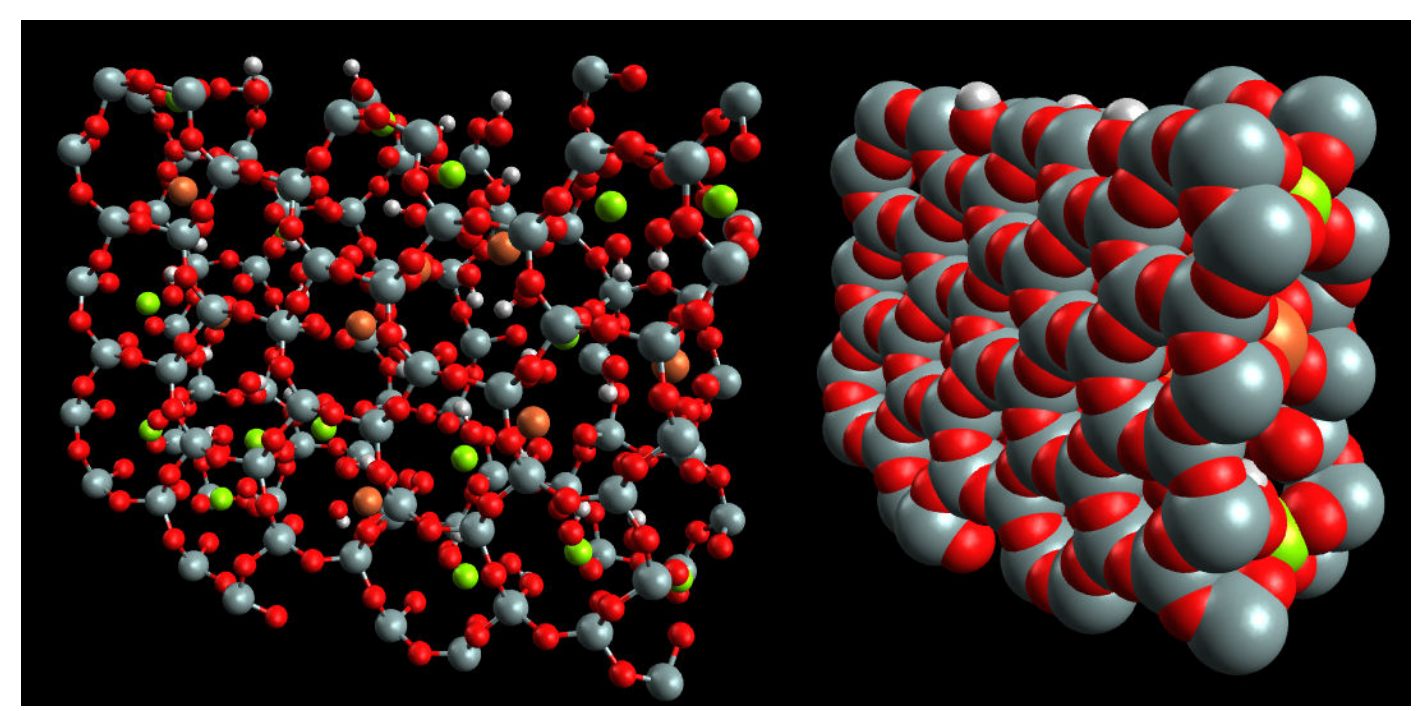

Fig. 3. Structure of the model (Avogadro 1.1.1) used in the simulations, optimized by MMFF94, visualized by ball-andstick model (left) and van der Waals spheres (right). The colors indicate the following atoms/ions: O (red), Si (blue), $\mathrm{Fe}^{3+}$ (orange), $\mathrm{Mg}^{2+}$ (green), $\mathrm{H}$ (white)

This structure is the result of the energy minimization, generated by the MMFF94 force field. The structure of the model does not contradict the previously published figures and it has the known physical properties. By comparison with the starting model, the geometry of this final structure is obviously different, the sheets of the crystal are both horizontally and vertically displaced. Considering the fact that montmorillonite is a mild and easily mouldable mineral, we can safely assume that this model represents the actual geometry better. It has an inner surface large enough to reproduce the molecular interactions in the interlaminar layer authentically. 


Journal of
Universal Science
Online

It is an important observation that the electrostatic interactions between the $\mathrm{Mg}^{2+} / \mathrm{Fe}^{3+} / \mathrm{OH}^{-}$ions and oxygens are primarily responsible for forming and stabilizing the structure and they have little influence on the mechanisms that take place in the interlaminar space. This can be explained by the surface charge conditions of the montmorillonite, visualized by Marvin Sketch (Fig. 4.).

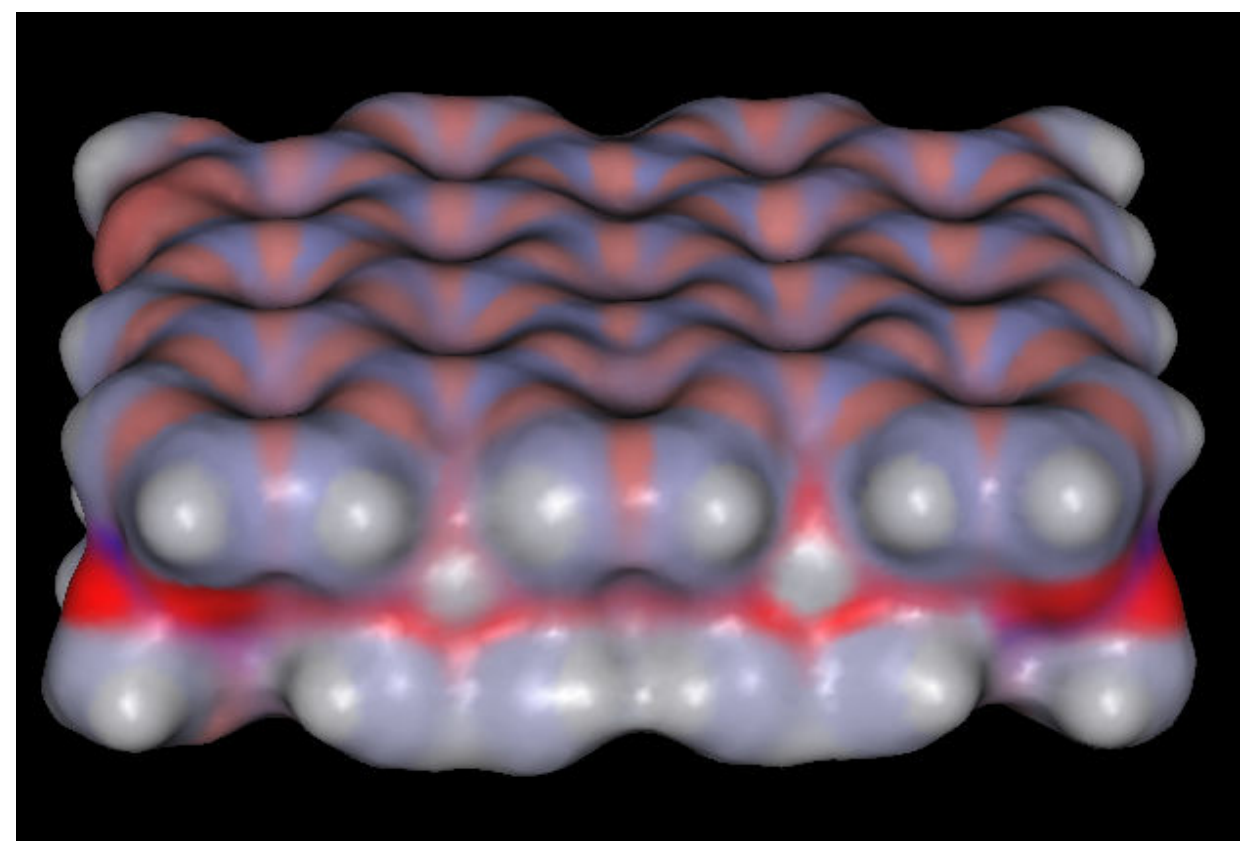

Fig. 4. Surface charge conditions of the montmorillonite layer optimized by MMFF94 (Marvin Sketch 14.8.18). Positive charges are blue, negative charges are red.

The positive (blue) and the negative (red) charges on the surface present a smooth, not intensely colored texture. The electrostatic fields of the ions within the octahedrons are shielded by the atoms of the tetrahedral sheets, therefore outside non electrostatic forces are more relevant, while in the interlaminar layer, electrostatic interactions dominate. The further studies (see below) have confirmed the hypothesis that the mechanisms that take place in the interlaminar layer are mostly affected by the charges of the tetrahedral sheets, if we consider only the montmorillonite's role in the adsorption of the DDT.

In the complete model (montmorillonite with pesticide molecules, water and $\mathrm{Na}^{+} / \mathrm{Ca}^{2+}$ in the interlaminar space) the water molecules were connected to each other and to some of the oxygens of the $\mathrm{Si}$ atoms by hydrogen bonds, so the model has the capability to chemically bind water. In order to create the hydrogen bond, the distance between the hydrogen and the oxygen of the Si-tetrahedral must be within 2 angstroms $(\AA)$, based on Avogadro's calculations. This bonds proved to be unstable, some of them existed during the optimization only for a short time, so we can assume that they can detach due to temperature change. The possible explanation to this phenomenon is that the oxygens in the tetrahedrons are connected with two Si-atoms that are larger in van der Waals radius, so the force field of the oxygen is partially shielded. Beside this, the electronegativity of the oxygen has a significantly higher value (3.5) than the $\mathrm{Si}$ (1.8), so there is a partially positive charge on $\mathrm{Si}$ and on the hydrogens of the water as well, therefore the proper orientation required for hydrogen bonds is energetically unfavorable.

An ion-induced dipole interaction occurred between the $\mathrm{Na}^{+} / \mathrm{Ca}^{2+}$ ions and the water molecules, as it was expected (see Fig. 5.). 


$\begin{aligned} & \text { Journal of } \\ & \text { Universal Science } \\ & \text { Online }\end{aligned} \quad \cdots, \cdots \cdots$

The DDT and the metabolites could not mix with the water due to the size and the hydrophobic character of the pesticide molecules. The water molecules and the cations showed a significant shift within the interlaminar space during the optimization time (some water molecules and ions got out of the interlaminar layer) but the pesticide molecules showed no appreciable movement. The explanation is that these hydrophobic molecules could not interact with the other components of the interlaminar layer, they have only a low partial charge on the chlorine atoms. The van der Waals forces of the two montmorillonite layers are also responsible for keeping the molecules in the interlaminar space. No difference was observed in the behavior of the DDD and DDE metabolites compared to DDT and the three-dimensional structures of the pesticide molecules were not changed during the optimization. One final state of the model after the optimization is shown in Fig. 5. The distance between the two layers is approximately $28.5 \AA$.

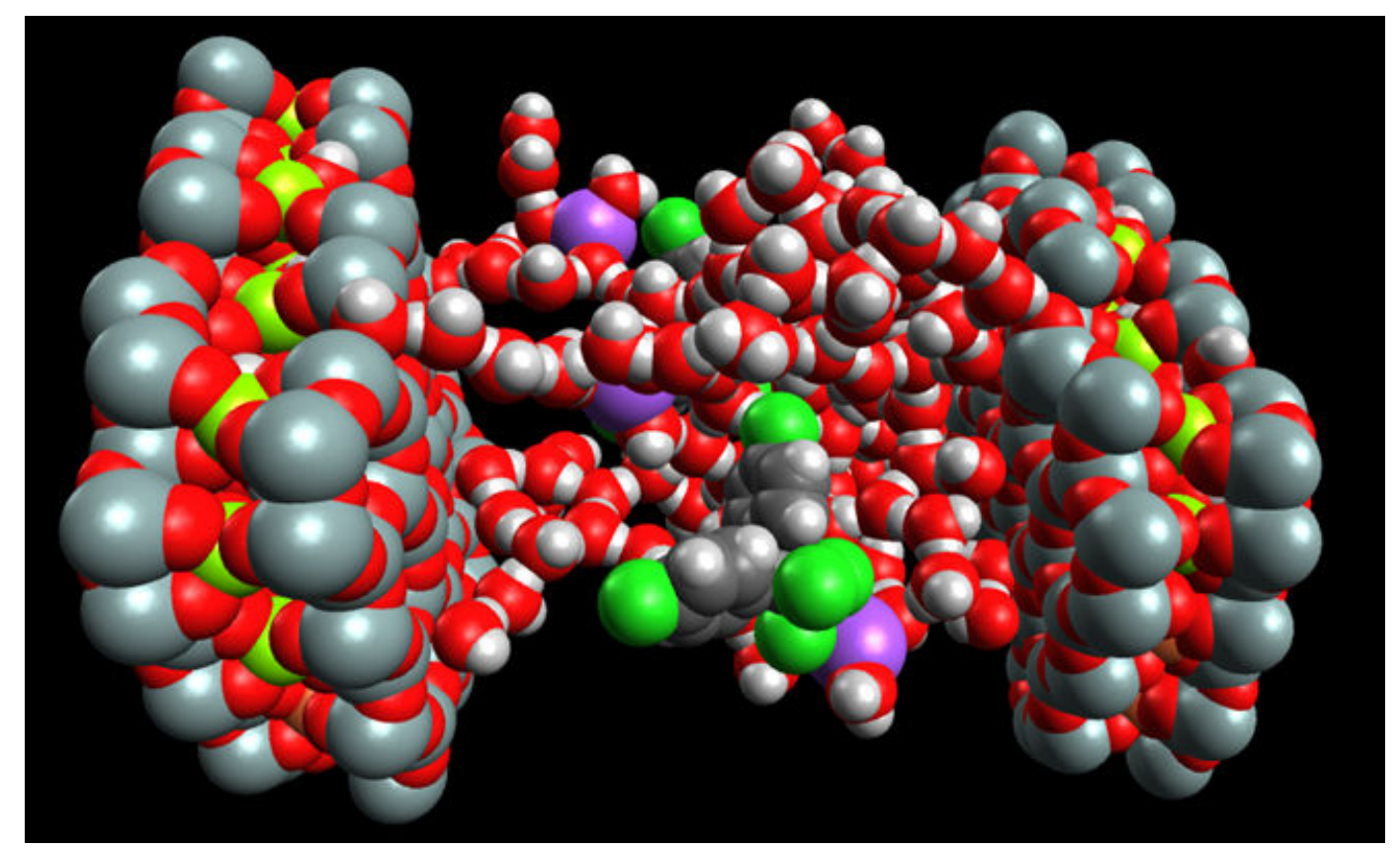

Fig. 5. The final state of the model after the MMFF94 optimization (Avogadro 1.1.1). Water molecules are connected by $\mathrm{H}$-bonds, $\mathrm{Na}^{+}$-s (purple) are hydrated but the DDT (with green chlorine atoms) is not dissolved in water and it's structure is unchanged.

\section{Conclusions}

The results of this short report reveal that the adsorption of the DDT and it's metabolites in the interlaminar space of the montmorillonite clay mineral is possible, primarily due to their hydrophobicity and the presence of van der Waals forces. This could only partly be responsible for the persistence of these insecticides. The complete model was a simplified structure that did not include many of the components that are present in real environmental conditions, e.g. humic substances which are the major organic constituents of soils and the examination of the surface adsorption of the pesticides was not part of this work.

With the use of a more complex model and other applications, it would be possible to understand the mechanism and dynamics of DDT's adsorption in more detail. 


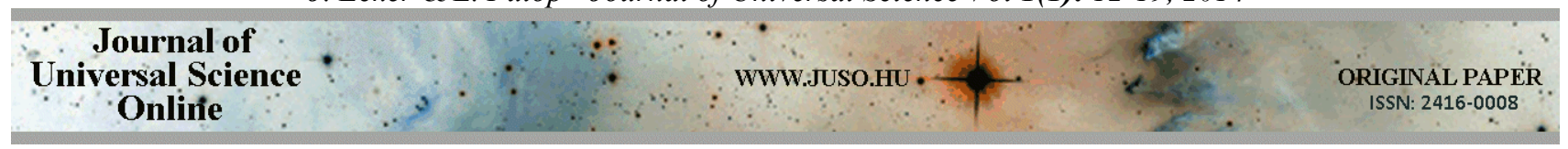

\section{References}

1. R. T. Baker, H. G. Applegate (1970) Effect of Temperature and Ultraviolet Radiation on the Persistence of Methyl Parathion and DDT in Soils. Agronomy Journal 62:(4) pp. 509-512

2. K. G. Bhattacharyya, S. S. Gupta (2008) Adsorption of a Few Heavy Metals on Natural and Modified Kaolinite and Montmorillonite: a Review. Advances in Colloid and Interface Science 140:(2) pp. 114-131

3. G. W. Brindley, G. Brown (1980) Crystal Structures of Clay Minerals and Their X-ray Identification. Minerological Society Monograph No. 5., London

4. D. Cheng, J. Yu, T. Wang, W. Chen, P. Guo (2014) Adsorption Characteristics and Mechanisms of Organochlorine Pesticide DDT on Farmland Soils. Polish Journal of Environmental Studies 23:(5) $p p$. $1527-1535$

5. S. Dousset, C. Mouvet, M. Schiavon (1994) Sorption of terbuthylazine and atrazine in relation to the physico-chemical properties of three soils. Chemosphere 28:(3) pp. 467-476

6. O. Faroon, M. O. Harris (2002) Chemical and Physical Information. In: Toxicological Profile for DDT/DDD/DDE. U. S. Department of Health and Human Services, Public Health Service, Agency for Toxic Substances and Disease Registry pp. 216

7. L. Fishbein (1974) Mutagens and potential mutagens in the biosphere I. DDT and its metabolites, polychlorinated biphenyls, chlorodioxins, polycyclic aromatic hydrocarbons, haloethers. Science of the Total Environment 2:(4) pp. 305-340

8. M. D. Hanwell, D. E. Curtis, D. C. Lonie, T. Vandermeersch, E. Zurek, G. R. Hutchison (2012) Avogadro: an advanced semantic chemical editor, visualization, and analysis platform. Journal of Cheminformatics 4:(17)

9. S. B. Hendricks (1941) Base Exchange of the Clay Mineral Montmorillonite for Organic Cations and its Dependence upon Adsorption due to van der Waals Forces. Journal of Physical Chemistry 45:(1) pp. 6581

10. S. Karaborni, B. Smit, W. Heidug, J. Urai, E. van Oort (1996) The Swelling of Clays: Molecular Simulation of the Hydration of Montmorillonite. Science 271:(5252) pp. 1102-1104
11. E. P. Lichtenstein, K. R. Schulz (1959) Persistence of Some Chlorinated Hydrocarbon Insecticides as Influenced by Soil Types, Rate of Application and Temperature. Journal of Economic Entomology 52:(1) $p p$. 124-131

12. S. Lin, R. Juang (2002) Heavy Metal Removal from Water by Sorption Using Surfactant-modified Montmorillonite. Journal of Hazardous Materials 92:(3) pp. 315-326

13. K. M. G. Machado, D. R. Matheus (2006) Biodegradation of Remazol Brilliant Blue $\mathrm{R}$ by Ligninolytic Enzymatic Complex Produced by Pleurotus ostreatus. Brazilian Journal of Microbiology 37: pp. 468-473

14. M. D. McGlamery, F. W. Slife (1966) The Adsorption and Desorption of Atrazine as Affected by $\mathrm{pH}$, Temperature and Concentration. Weeds 14:(3) pp. 237-239

15. G. C. Miller, R. G. Zepp (1979) Photoreactivity of Aquatic Pollutants Sorbed on Suspended Sediments. Environmental Science \& Technology 13:(7) $p p$. 860-863

16. B. Ngabe, T. F. Bidleman (2006) DDT Concentrations in Soils of Brazzaville, Congo. Bulletin of Environmental Contamination and Toxicology 76:(4) pp. 697-704

17. L. Pauling (1930) The Structure of Micas and Related Minerals. Proceedings of the National Academy of Sciences 16: $p p$. 123-129

18. M. Ricking, J. Schwarzbauer (2012) DDT Isomers and Metabolites in the Environment: an Overview. Environmental Chemistry Letters 10:(4) pp. 317-323

19. J. Sternburg, C. W. Kearns, H. Moorefield (1954) Resistance to DDT, DDT-Dehydrochlorinase, an Enzime Found in DDT-Resistant Flies. Journal of Agricultural and Food Chemistry 2:(22) pp. 11251130

20. J. E. Thomas, L. T. Ou, A. All-Agely (2008) DDE Remediation and Degradation. Reviews of Environmental Contamination and Toxicology 194: pp. 55-69 


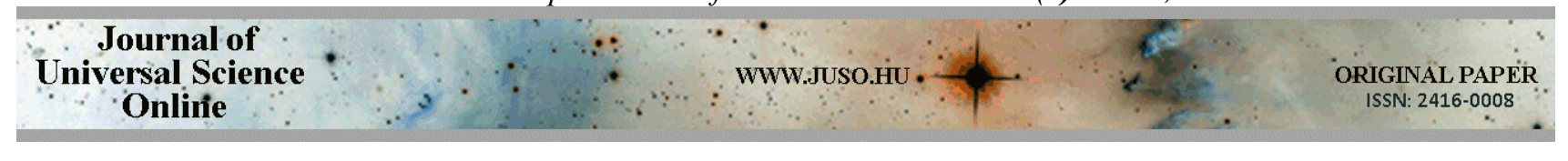

21. F. Wang, X. Jiang, Y. Bian, F. Yao, H. Gao, G. Yu, J. C. Munch, R. Schroll (2007) Organochlorine pesticides in soils under different land usage in the Taihu Lake region, China. Journal of Environmental Sciences 19:(5) pp. 584-590

22. F. Wania, D. MacKay (1996) Peer Reviewed: Tracking the Distribution of Persistent Organic
Pollutants. Environmental Science and Technology 30:(9) pp. 390-396

23. G. M. Woodwell, R. T. Martin (1964) Persistence of DDT in Soils of Heavily Sprayed Forest Stands. Science 145:(3631) pp. 481-483 